\title{
ANÁLISE DO FENÓMENO DE BASE SEGURA EM CONTEXTO FAMILIAR: AS RELAÇÕES CRIANÇA/MÃE E CRIANÇA/PAI ${ }^{1}$
}

\author{
Ligia Monteiro ${ }^{2}$ \\ Manuela Veríssimo 3 \\ Brian E. Vaughn ${ }^{4}$ \\ António J. Santos ${ }^{5}$ \\ Marília Fernandes 6
}

Resumo: O estudo analisa, no contexto da teoria de Bowlby e Ainsworth, o modo como a criança utiliza as figuras parentais como base segura, explorando a especificidade da organização destes comportamentos nas duas relações. Analisa, ainda, a existência de semelhanças ou diferenças nas classificações da criança em relação à mãe e ao pai. Os participantes são 56 díades mãe/criança e pai/criança, tendo as crianças idades compreendidas entre os 29 e os 38 meses. Observadores independentes realizaram visitas domiciliárias com a criança/mãe e com a criança/pai, utilizando o $A Q S$ (Waters, 1995). Não foram encontradas diferenças significativas entre os valores de segurança para a mãe e para o pai. Com base nas escalas do $A Q S$, obtiveram-se diferenças significativas na Proximidade e no Contacto Físico. Assim, a criança utiliza ambos os pais como base segura, embora os estilos de interacção possam apresentar características específicas. Verificou-se, ainda, a existência de uma correlação significativa entre os valores de segurança da criança em relação à mãe e ao pai, o que vai ao encontro dos resultados obtidos por Veríssimo et al. (2006). Esta correlação poderá ser explicada pela semelhança nos cuidados parentais.

\footnotetext{
${ }^{1}$ Contacto: Lígia Monteiro, Instituto Superior de Psicologia Aplicada, Rua Jardim do Tabaco, 34, 1100 Lisboa. Lígia_monteiro@ispa.pt.

2 Doutoranda Universidade Nova/ISPA, Bolseira F.C.T. (SFRH/BD/10277/2002), UIPCDE.

3 Professora Associada, ISPA, UIPCDE.

${ }^{4}$ Human Development and Family Studies, Auburn University.

5 Professor Associado, ISPA, UIPCDE.

${ }^{6}$ Discentes da área de Psicologia Educacional, ISPA.

Agradecimentos: os autores gostariam de agradecer a todas as mães, pais e crianças que aceitaram participar neste estudo, financiado pela FCT (POCTI, Plurianual, UIPCDE). Os autores gostariam, ainda, de agradecer a colaboração de André Silva, Iolanda Queiroz, Ana Zilda Silva, Carla Oliveira, Inês Peceguina, Vera Oliveira e Sofia Meneres, na recolha dos dados. Os autores gostariam, também, de agradecer os comentários valiosos da professora Kelly Bost e da professora Zilda Fidalgo.
}

PSICOLOGIA, Vol. XXII (1), 2008, Edições Colibri, Lisboa, pp. 105-125. 
Palavras-chave: fenómeno de base segura, figuras parentais, semelhanças vs. diferenças nas relações.

The study of the secure base phenomenon in the family context: child/mother and child/father relationships (Abstract): This paper high lights the importance of a family system approach to the study of attachment, in a naturalistic environment, by analysing the way the child uses both mother and father as a secure base, as well as, the concurrence or independence of this attachment relationships. 56 mother/child and father/child díades participated in the study. Children's ages range between 29 and 38 months. Independent observers, made home observations of the child with the mother and the child with the father, using the AQS (Waters, 1995). No differences were found between mean averages for security scores for mothers and fathers, showing that the child is able to use both parents as secure-bases. Significant differences were found in the Proximity and Physical Contact scales between both parents, suggesting that mothers and fathers probably have different communicative styles. A significant correlation was found between security scores for mother and father, a result comparable to the one obtained by Veríssimo et al. (2006). This could be explained by the similarity on parental caregiving behaviour.

Keywords: secure base phenomenon, parental figures, similarities vs. difference.

\section{Introdução}

No contexto da teoria da vinculação, a mãe tem sido considerada a figura principal (Bowlby, 1982, 1989; Ainsworth, 1989; Ainsworth, Blehar, Waters \& Wall, 1978), pelo que, até há poucos anos, a investigação realizada na área centrou-se, essencialmente, na relação mãe/criança. Contudo, no contexto familiar, a criança pode interagir, de um modo contínuo e estável, com outras figuras significativas, como o pai, os irmãos ou mesmo os avós (Thompson, 1998; Sagi, van IJzendoorn, Aviezer, Donnell, Koren-Karie, Joels \& Harel, 1995). Apesar dos enormes progressos realizados na compreensão da relação mãe/criança, há ainda um longo caminho a percorrer no estudo da vinculação no micro-sistema das relações familiares (Bretherton, 1992), particularmente num período em que a configuração tradicional da família tem sofrido profundas transformações, conduzindo a uma redefinição dos papéis parentais (Cabrera, Tamis-LeMonda, Bradley, Hofferth \& Lamb, 2001). Assim, o estudo do modelo do sistema familiar, em vez de, apenas, o modelo mãe/criança, pode permitir uma compreensão mais diferenciada e integrada dos antecedentes e das consequências dos diferentes tipos de vinculação (Cowan, 1997).

A relação de vinculação é definida, num contexto etológico e evolutivo, como um forte laço afectivo que se estabelece por volta dos 7/8 meses 
(Bowlby, 1982) e que liga a criança a uma ou mais figuras estáveis na sua vida, tidas como únicas, ao longo do tempo e dos contextos. O sistema que regula esta relação é definido como um sistema de controlo comportamental, que funciona com o objectivo de manter o equilíbrio entre os comportamentos de vinculação e de exploração do meio. Em contextos familiares, e na ausência do que Bowlby (1973) designa por "pistas naturais de perigo", verifica-se o favorecimento dos comportamentos de exploração. A criança afasta-se da mãe, regressando para junto dela, periodicamente, por sua iniciativa, para brincar ou para estabelecer um breve contacto, afastando-se novamente para explorar os objectos ou interagir com outras pessoas (Ainsworth et al., 1967; Ainsworth et al., 1978; Bowlby, 1982, 1989). Noutros contextos que podem suscitar perigo ou que podem estar associados a experiências negativas, a tendência será favorecer os comportamentos de proximidade e o contacto físico com a mãe, assistindo-se a um declínio dos comportamentos de exploração (Ainsworth et al., 1978; Bowlby, 1982, 1989). O funcionamento do sistema de controlo de vinculação é definido como "fenómeno de base segura" (Waters \& Deane, 1985; Waters \& Cummings, 2000), influenciando a organização dos afectos, das cognições e dos comportamentos nas relações de vinculação durante a vida. Esta relação vai sendo construída, ao longo do desenvolvimento, no contexto das interacções únicas estabelecidas entre a criança e a figura de vinculação, onde a qualidade dos cuidados parentais (em particular, a sensibilidade, responsividade e a acessibilidade) é tida como fundamental para uma organização segura ou insegura (ambivalente ou evitante) dos comportamentos (Ainsworth, 1989; Ainsworth et al., 1978). Segundo Bowlby, os elementos centrais desta relação vão-se organizando numa representação interna à medida que a criança e a relação se desenvolvem. Quando a história das experiências durante a infância, com a figura de vinculação, é marcada pela sensibilidade, responsividade e acessibilidade, provavelmente, a criança constrói um modelo dessa figura como responsivo, acessível, e desenvolverá um modelo de self correspondente, como valorizado ou merecedor de afecto e cuidados. Se a criança vivencia trocas frustrantes, envolvendo insensibilidade ou rejeição, provavelmente irá construir modelos internos negativos da figura de vinculação e do self. Estas diferenças a nível cognitivo (expectativas face ao comportamento materno) conduzem, assim, a diferentes respostas por parte da criança, quer em situações de separação ou de stress, quer de exploração do meio (Ainsworth et al., 1978; Bowlby, 1973).

Bowlby (1982) refere que, durante o segundo e a maior parte do terceiro ano de vida, o comportamento de vinculação não é exibido com menor intensidade ou frequência do que no primeiro ano. Contudo, a natureza das circunstâncias que o activam muda, resultado da rápida expansão do mundo físico e social, pelo que o uso de figuras de vinculação como base segura se 
mantém central na organização do comportamento da criança, para a sua sobrevivência e socialização. Os estudos, segundo Marvin e Britner (1999), sobre as mudanças no desenvolvimento do sistema de vinculação sugerem que, do primeiro até por volta do terceiro ano de vida, a organização dos comportamentos da criança face à figura de vinculação é sensivelmente a mesma. Apesar de algumas mudanças em termos de desenvolvimento, a manutenção de um grau razoável de proximidade permanece como o maior objectivo da criança em relação à mãe. Por volta do terceiro ano assiste-se a uma complexificação progressiva dos modelos internos dinâmicos do self e da mãe, resultado da aquisição de novas competências cognitivas e comunicativas, o que conduzirá, por volta do quarto ano, a uma relação de parceria, que Bowlby (1982) designou de "parceria corrigida para a meta". A criança é capaz de operar internamente e simultaneamente sobre a sua perspectiva e a da figura de vinculação, integrando-as, sendo capaz de manter a relação orientada para um fim, baseada em objectivos, planos e sentimentos partilhados. Deste modo, o objectivo final da relação de vinculação passará do contacto e da proximidade física para a disponibilidade da figura de vinculação (grau em que esta figura está, na perspectiva da criança, acessível e responsiva aos seus sinais e comunicações), se por qualquer razão a criança o desejar (Bowlby, 1973; Marvin \& Britner, 1999). O objectivo do sistema comportamental da vinculação ao longo do desenvolvimento será "sentir-se seguro" (Sroufe \& Waters, 1977).

Tanto Bowlby (1982) como Ainsworth (1967) reconhecem que a maioria das crianças no final do primeiro ano se encontram vinculadas a outras figuras, para além da mãe, nomeadamente, ao pai. Lamb, Frodi, Hwang e Frodi (1983) referem diversos estudos realizados em situações estruturadas e não estruturadas, nos quais se verificou que as crianças formam laços vinculativos a ambos os pais por volta da mesma idade. No entanto, a figura paterna tem sido vista como uma figura de vinculação num contexto temporal e ecológico diferente, bem como secundário, relativamente à mãe (Grossmann, Grossmann, Fremmer-Bombik, Kindler, Scheuerer-Englisch \& Zimmermann, 2002). Segundo Ainworth (1989), “... the tendency has been to consider the bond of the father to child as somehow less deeply rooted than the bond of mother to child" (p. 712). Bowlby (1982) assume que existe a tendência de preferir uma figura principal na procura de segurança e conforto, pelo que as figuras de vinculação não são todas tratadas de modo igual pela criança, existindo, assim, uma hierarquia. Para o autor, a mãe é vista, usualmente, como a principal figura de vinculação, sendo o pai visto, essencialmente, como um companheiro de brincadei$\mathrm{ra}$, ao qual as crianças dirigem, preferencialmente, comportamentos de carácter afiliativo. O pai exerce, ainda, um importante papel de suporte finan- 
ceiro e de apoio emocional à mãe, ajudando-a a manter um clima harmonioso e propício ao desenvolvimento do bebé (Bowlby, 2002).

Observações realizadas por Lamb (1977) revelam que, em situações onde não existem elementos de stress, nomeadamente, em casa, as crianças, durante $01 .^{\circ}$ ano de vida, não apresentam preferência por nenhum dos pais na exibição de comportamentos vinculativos e face a estranhos optam sempre pelas figuras parentais. Contrariamente, Lamb et al. (1983) verificaram uma preferência pela figura materna na orientação dos comportamentos de vinculação aos 8 e 16 meses. Em acontecimentos onde elementos de stress crescente estão presentes, a criança tende a dirigir os comportamentos de vinculação à figura que estiver presente, em detrimento de estranhos. $\mathrm{Na}$ presença de ambos os pais, a criança procura, com maior probabilidade, o conforto da mãe (Ainsworth, 1967; Lamb, 1976, cit. por Lamb et al., 1983).

Embora no geral se encontrem semelhanças, na realidade existem diferenças, empiricamente documentadas, entre a natureza e o estilo de comportamentos maternos e paternos na interacção com a criança (Lewis \& Lamb, 2003). Para Cox, Owen, Henderson e Margand (1992), as origens da segurança da vinculação pai/criança podem residir em diferentes tipos de interacção. Apesar da tendência para um maior envolvimento, comparativamente com as mães, os pais passam menos tempo com os filhos, sendo que as interacções com as mães envolvem, essencialmente, prestação de cuidados, enquanto que os pais passam, proporcionalmente, mais tempo de interacção em brincadeira, pelo que estas duas relações envolvem diferentes tipos de experiências para a criança (Lamb, 1987). Porém, os pais são tão capazes como as mães de cuidar dos seus filhos, sendo competentes e sensíveis nas interacções com os mesmos, parecendo que à medida que as crianças se desenvolvem e os pais se tornam mais experientes, estes se vão sentindo mais à-vontade no seu papel, embora os estilos sejam diferentes (Belsky, Gilstrap \& Rovine, 1984).

A meta-análise realizada por van IJzendoorn e De Wolff (1997) revelou uma associação entre sensibilidade paterna e segurança da vinculação criança/pai que, contudo, é mais fraca $(r=0,13)$ do que a associação entre sensibilidade materna e vinculação criança/mãe $(r=0,24)$. Alguns autores (e.g., Cox et al., 1992; Goossens \& van IJzendoorn, 1990) propõem a análise da interacção criança/pai, também, num contexto de brincadeira, bem como o seu alargamento a outras dimensões do comportamento parental. Cox et al. (1992) sugerem, por exemplo: a brincadeira recíproca, o nível de actividade, os afectos físicos ou o encorajamento adequado no sentido do sucesso, considerando o seu compósito mais eficaz na previsão da relação de vinculação da criança ao pai. Salientam, também, a importância das atitudes paternas face à criança e ao seu papel enquanto pai. Para Grossmann et al. (2002), a sensibilidade no contexto de brincadeira, por parte do pai, é central na relação de vinculação criança/pai, do mesmo modo que a sensibilidade na prestação de cuidados é central na relação 
criança/mãe, uma vez que parece ser nestes domínios que estas figuras são mais salientes.

Uma das questões centrais no estudo das diferentes relações de vinculação estabelecidas pela criança refere-se às semelhanças versus diferenças na qualidade das relações. Os resultados obtidos divergem. Vários estudos (e.g., Bridges, Connell \& Besky, 1988; Cox et al., 1992; Main \& Weston, 1981) verificaram a existência de diferenças nas classificações da qualidade de vinculação da criança à mãe e ao pai. O que vai ao encontro da noção de que a segurança da vinculação é específica e reflecte a história de interacções únicas estabelecidas entre a figura parental e a criança. Ou seja, a segurança ou insegurança é característica da relação e não é um traço da criança (Ainsworth et al., 1978; Bowlby, 1982; Main \& Weston, 1981; Sroufe \& Waters, 1977; Sroufe, 1985).

Na meta-análise realizada por van IJzendoorn e De Wolff (1997) encontrou-se apenas uma associação modesta $(r=0,17)$ entre a qualidade da vinculação da criança à mãe e ao pai, considerando os autores que a segurança da vinculação não parece estar, substancialmente, generalizada às diferentes relações dentro do sistema familiar. Outros estudos (e.g., Caldera, 2004; Fox, Kimmerly \& Schafer, 1991, Frosch, Mangelsdorf \& McHale, 2000; Rosen \& Rothbaum, 1993; Rosen \& Burke, 1999; Steele, Steele \& Fonagy, 1996; Veríssimo, Monteiro \& Santos, 2006) verificaram a semelhança ou concordância das classificações em relação a ambas as figuras parentais. Estes resultados podem reflectir a influência da relação criança/mãe nos comportamentos da criança com outras figuras, nomeadamente, com o pai (Steele et al., 1996), ou o impacto de características individuais da criança, nomeadamente, o temperamento (Fox et al., 1991; Cassidy, 1999) na relação de vinculação. Podem, ainda, ser resultado da semelhança nos cuidados parentais e nos seus sistemas de valores relativos a assuntos importantes para a segurança da vinculação, como a sensibilidade e a responsividade aos pedidos e necessidades da criança. Se tanto a mãe, como o pai são sensíveis e responsivos aos sinais da criança, será de esperar um elevado grau de concordância nas classificações da criança a ambos (Cassidy, 1999; Fox et al., 1991). Um progenitor poderá, inclusivamente, servir de modelo ao outro ou ambos poderão aprender em conjunto a responderem sensivelmente (ou insensivelmente) à criança (Belsky, Rovine \& Taylor, 1984; Fox et al., 1991; Sagi et al., 1995).

A maioria dos estudos empíricos realizados, no contexto da teoria da vinculação, situa-se na primeira infância, utiliza como instrumento de avaliação a Situação Estranha (Ainsworth et al., 1978) e analisa, essencialmente, a díade mãe/criança. À medida que o estudo da vinculação ultrapassa a infância e se recorre a medidas que implicam a representação mental, há que não perder o enfoque no comportamento, uma vez que a ligação 
entre modelo interno dinâmico e comportamento é central na teoria de Bowlby, dado que a função destes modelos é a de organizar o comportamento de um modo mais flexível (Marvin \& Britner, 1999). Sendo os anos pré-escolares caracterizados por importantes desenvolvimentos a nível cognitivo, linguístico, motor e social, mas durante os quais os comportamentos de vinculação permanecem centrais na organização do comportamento da criança (Marvin \& Britner, 1999), será fundamental a sua análise, quer na relação com a figura materna, quer paterna. Este parece ser, também, um período de crescente interesse e envolvimento nas interacções pai/criança (Easterbrooks \& Goldberg, 1984, Lamb, 1977).

Um dos instrumentos de avaliação utilizado na análise dos comportamentos de base segura é o Attachment Behaviour Q-Set (Waters, 1995). A validade do $A Q S$, realizado por observadores, foi claramente confirmada na meta-análise de van IJzendoorn, Vereijken, Bakermans-Kranenburg e Riksen-Walraven (2004), que incluem esta medida de avaliação da vinculação na mesma categoria, em termos de qualidade, da Situação Estranha e do Adult Attachment Interview. Das 139 amostras incluídas nesta meta-análise, 80 são oriundas do continente norte-americano e em, apenas, 10 a relação da criança ao pai é analisada. Destas, 9 utilizam o AQS self-report. A escassez de estudos relativos ao pai levou a que, de uma perspectiva meta-analítica, a validade do $A Q S$ para a figura paterna tenha, ainda, que ser documentada. As idades das crianças participantes nestas amostras variam entre os 12 e os 56 meses.

O presente estudo procura ser um contributo para a teoria da vinculação ao alargar a sua análise à díade pai/criança, na faixa dos 2 anos e meio/3 anos, procurando compreender o modo como a criança utiliza as figuras parentais como base segura, em contexto naturalista, nomeadamente, em casa, onde o fenómeno se desenvolve e é moldado (Waters, Vaughn, Posada \& Kondo-Ikemura, 1995). Este estudo visa, ainda, explorar a especificidade da organização dos comportamentos de base segura da criança face às figuras parentais, procurando compreender melhor as características destas relações. Por último, analisa a existência de semelhanças ou diferenças nas classificações das relações de vinculação da criança à mãe e ao pai, utilizando o $A Q S$ (Waters, 1995) realizado por observadores.

\section{Método}

\section{Participantes}

Os participantes são 56 díades mãe/criança e pai/criança. À data das observações do A.Q.S., as crianças tinham idades compreendidas entre os 29 e os 38 meses $(M=31,91 ; D P=2,56)$ sendo 29 do sexo feminino e 27 do 
sexo masculino, todas caucasianas. As crianças são provenientes de famílias biparentais, à excepção de 2 crianças que vivem com as mães, tendo, contudo, um contacto regular com os pais. Destas, 28 são filhos primogénitos e 39 têm irmãos. A sua idade de entrada na creche/jardim-de-infância varia entre os 4 e os 30 meses $(M=8,67 ; D P=6,59)$ passando entre 3 a 10 horas $(M=7,56 ; D P=1,53)$ na mesma. As mães tinham idades entre os 26 e 48 anos $(M=34,95 ; D P=4,33)$ e os pais entre os 28 e os 63 anos $(M=37,48$; $D P=6,08)$. As habilitações literárias das mães variam entre os 7 e os 23 anos de escolaridade $(M=15,46 ; D P=3,34)$ e as dos pais entre os 7 e os 23 anos $(M=14,77 ; D P=3,17) .51$ das mães trabalham fora de casa, assim como 55 dos pais. As famílias pertencem a um nível socio-económico médio e médio alto, tendo sido recrutadas para o projecto através das creches e jardins-de-infância de ensino particular que as crianças frequentam. Os participantes fazem parte de um projecto mais vasto: Longitudinal Study of Secure Base Relationships: Behavioral Organization and Mental Representations, coordenado pela Prof. Manuela Veríssimo e pelo Prof. António J. Santos, que analisa o desenvolvimento emocional e social das crianças entre os 2 anos e meio e os 5 anos.

\section{Instrumentos}

Attachment Behaviour Q-Set (AQS) - versão 3.0 de Waters (1995)

O $A Q S$ avalia a qualidade do comportamento de base segura da criança, face à mãe ou a outras figuras, em contexto ecologicamente válido. No $A Q S$, o comportamento de base segura é definido como a organização harmoniosa e o equilíbrio adequado entre a procura de proximidade e a exploração do meio (Posada, Goa et al., 1995). O instrumento permite a descrição pormenorizada do comportamento vinculativo da criança e do seu desenvolvimento, possibilitando a observação das mudanças e continuidades individuais no mesmo (Veríssimo, Blicharski, Strayer \& Santos, 1995).

Este é um instrumento de classificação de observação sistemática, baseada na metodologia do Q-Sort, a qual permite obter uma distribuição quase normal dos dados (Waters, Noyes, Vaughn \& Ricks, 1985). Tal como a maioria dos $Q$-Sort, o $A Q S$ é preenchido através da atribuição de itens a categorias, utilizando uma distribuição fixa. Os 90 itens do instrumento são distribuídos pelo observador numa escala de nove níveis que variam do "extremamente característico" até ao "extremamente incaracterístico". Esta distribuição deve ser realizada tendo em consideração a sua saliência ou relevância para a criança a ser descrita. $\mathrm{O}$ valor de critério é o resultado de um composto de sorts de especialistas referente ao constructo em causa (Waters \& Deane 1985). Deste modo, as descrições de crianças individuais podem ser comparadas com o valor de critério de "segurança" da criança 
ideal, obtendo-se um valor que reflecte o grau de congruência entre o indivíduo e o critério da "criança ideal". Este representa o lugar ocupado pela criança num contínuo, sendo que as crianças mais capazes de utilizar a mãe, ou outra figura, como base segura recebem valores mais elevados, enquanto que as menos capazes de o fazer recebem valores mais baixos. A existência do fenómeno de base segura não é assumida por esta técnica, antes a sua presença ou ausência é inferida do perfil (Posada, Goa et al., 1995).

\section{Escalas de Posada e Waters (1995)}

As Escalas de Posada e Waters (1995, cit. por Posada, Waters, Crowell \& Lay, 1995) são derivadas do $A Q S$. Os itens seleccionados do $A Q S$ para as diferentes escalas foram os que conceptualmente se encontravam relacionados com o constructo de base segura e que poderiam ser observados nas visitas domiciliárias. Estes itens incluem comportamentos centrais para o fenómeno de base-segura, comportamentos da criança quando interagindo com a sua mãe/pai e comportamentos com outros adultos mediados pela mãe/pai. As quatro escalas referem-se a domínios específicos do comportamento da criança: Interacção Suave com a Mãe/Pai; Proximidade com a Mãe/Pai; Contacto Físico com a Mãe/Pai e Interacção com outros Adultos (Veríssimo et al., 2006). Os alphas de Cronbach obtidos nas 4 escalas para a mãe variam entre 0,$87 ; 0,90 ; 0,83 ; 0,78$, respectivamente, e para o pai variam entre 0,$86 ; 0,92 ; 0,84 ; 0,78$. Estes valores traduzem a elevada fiabilidade das escalas para ambas as figuram parentais e são comparáveis aos obtidos por Posada, Waters et al. (1995).

\section{Procedimento}

\section{Observações do AQS}

As visitas domiciliárias, de cerca de 2 a 3 horas, foram combinadas com cada um dos pais, num momento do dia em que estivessem disponíveis, com vista a observar a interacção da criança com a mãe/pai. Aquando da observação da díade mãe/criança, foi pedido que o pai não estivesse presente, tendo o mesmo sido solicitado à mãe aquando da observação da díade pai/criança. As visitas foram realizadas obedecendo ao princípio de contrabalanceamento, de modo a controlar possíveis efeitos de ordem. O intervalo das visitas foi, em média, de 1 mês. Cerca de $82 \%$ das visitas, no caso das mães, e $64 \%$, no caso dos pais, foram realizadas durante a semana, após os pais terem ido buscar as crianças à escola, as restantes visitas foram realizadas no fim-de-semana.

Foi dito aos pais que o objectivo da visita era conhecer a criança e a mãe/pai na sua rotina e experiências diárias, pelo que lhes foi pedido que mantivessem as suas actividades do dia-a-dia sem alterações devido à pre- 
sença dos observadores. Não foram colocadas quaisquer restrições às díades aquando das observações. Estas foram realizadas por dois observadores que se comportaram como se de visitas sociais da casa se tratassem, procurando não interferir na rotina familiar, mas participando das brincadeiras da criança quando solicitados e conversando informalmente com a mãe/pai, tendo sempre o cuidado de não perturbar as interacções. Quando se tornava oportuno, e na sequência da conversa com a mãe/pai, foram-lhes colocadas questões acerca de itens que não se podem observar (e.g., o item 10 refere-se ao comportamento da criança quando se vai deitar) e de itens que não foram observados na visita (e.g., o item 45 refere-se ao facto de a criança gostar de cantar e dançar ao som de músicas).

Os dois observadores que realizaram as visitas domiciliares às mães não foram os mesmos que efectuaram as visitas aos pais. No final de cada observação, os observadores realizaram a distribuição dos itens do $A Q S$ relativos ao sujeito, de modo totalmente independente (Veríssimo, Monteiro, Vaughn, Santos \& Waters, 2005).

Os observadores foram treinados durante um período de várias semanas antes de iniciarem as observações, tendo discutido os itens e completado o Q-Sort da criança idealmente segura em conjunto com a Coordenadora do Projecto, como parte do treino. Antes do início da recolha dos dados obteve-se uma concordância interobservadores entre 0,60-0,89. Para as observações realizadas nas díades em estudo, a concordância obtida foi em média de 0,72 para as mães e de 0,73 para os pais. O Q-Sort de cada criança foi, assim, obtido pela média das duas descrições $Q$ realizadas pelos observadores.

\section{Resultados}

\section{Qualidade da vinculação da criança à mãe e ao pai}

$\mathrm{O}$ valor de segurança da criança em relação à mãe e ao pai foi obtido utilizando o valor do critério de segurança do $A Q S$ de Waters (1995). Convencionou-se que o valor de segurança da criança é a correlação de Pearson entre o valor do critério de segurança da criança idealmente segura e o $Q$ -sort individual da criança. Os resultados para a mãe e para o pai mostram poucos valores de segurança negativos ou zero, o que, de acordo com Posada, Goa et al. (1995), indica que a criança utiliza quer a mãe, quer o pai como base segura. $O$ valor de segurança para a mãe varia entre $-0,12$ e 0,79 com uma média de $0,45(D P=0,21)$. Para o pai os valores variam entre $-0,04$ e 0,79 com uma média de $0,41(D P=0,20)$.

Os valores médios obtidos na meta-análise de van IJzendoorn et al. (2004) para o valor de segurança em relação à mãe variam entre 0,25 e 0,65 
e para o pai entre 0,29 e 0,44, em amostras não clínicas. Uma análise de variância revelou que não existem diferenças significativas entre os valores de segurança da criança, na relação com a mãe e o pai $(F(110,1)=1,27$; $p>0,05)$ ou seja, as crianças utilizam ambos como base segura.

\section{Análise dos descritivos}

Com base no Coeficiente de Correlação de Pearson (R), analisaram-se as relações entre as variáveis descritivas e o valor de segurança das crianças relativamente à mãe e ao pai.

Tabela 1: Correlações entre as variáveis descritivas e os valores de segurança para mães e pais

\begin{tabular}{lcc}
\hline & Segurança/mãe & Segurança/pai \\
\cline { 2 - 3 } Idade/criança & $-0,22$ & $-0,02$ \\
Idade entrada creche/j.j.i. & $-0,11$ & $-0,21$ \\
N. ${ }^{\text {horas creche/j.-i. }}$ & $-0,13$ & 0,07 \\
Idade/mãe & $-0,25$ & $-0,07$ \\
Idade/pai & $*-0,32$ & $-0,06$ \\
Hab. Literárias/mãe & $-0,08$ & $-0,12$ \\
Hab. Literárias/pai & 0,13 & $-0,00$ \\
\hline
\end{tabular}

Nota: * A correlação é significativa a $p<0,05$.

De acordo com a teoria da vinculação, não seria de esperar a existência de diferenças em função da idade ou habilitações literárias da mãe e do pai. Contudo, como se pode observar, na Tabela 1, foi encontrada uma correlação negativa, significativa, entre a idade do pai e o valor de segurança da criança em relação à mãe, ou seja, quanto mais elevada a idade do pai, mais baixo o valor de segurança relativamente à mãe. Quanto à idade de entrada na creche/jardim-de-infância e ao número de horas que as crianças aí passam, os dados vão ao encontro dos resultados obtidos no estudo NICHD (1997) que referem não serem estas variáveis per se que explicam as diferenças na qualidade da vinculação da criança à mãe.

Com base numa análise de variância, não foram encontradas diferenças significativas entre os valores de segurança em relação à mãe para as raparigas $(M=0,44 ; D P=0,22)$ e rapazes $(M=0,46 ; D P=0,20)(F(54,1)=$ $0,15, p>0,05)$; nem entre os valores de segurança relativamente ao pai para as raparigas $(M=0,38 ; D P=0,21)$ e rapazes $(M=0,44 ; D P=0,18)$ $(F(54,1)=0,92, p>0,05)$. Analisando a ordem de nascimento, verificou-se 
que não existem diferenças significativas entre os valores de segurança à mãe nas crianças primogénitas $(M=0,46 ; D P=0,21)$ e não primogénitas $(M=0,45 ; D P=0,22)(F(53,1)=0,019, p>0,05)$ nem entre os valores de segurança ao pai nas crianças primogénitas $(M=0,45 ; D P=0,22)$ e não primogénitas $(M=0,37 ; D P=0,18)(F(53,1)=2,10, p>0,05)$. Assim, tal como se verificou noutros estudos (e.g. Caldera, 2004; Frosch et al., 2000; Main \& Weston, 1981), o género e ordem de nascimento da criança não estão associados à segurança, quer para a mãe, quer para o pai.

\section{Comportamentos da criança na interacção com a mãe e o pai}

Com vista a melhor compreender o modo como a criança organiza os seus comportamentos de base segura face à mãe e ao pai, utilizaram-se as quatro escalas do $A Q S$ de Posada e Waters (1995 cit. por Posada, Waters et al., 1995). As médias e os desvios-padrão são apresentados na Tabela 2.

Tabela 2: Médias e desvio-padrão das escalas do A.Q.S. para mãe e pai

\begin{tabular}{lcccc}
\hline \multirow{2}{*}{ Escalas } & \multicolumn{2}{c}{ Mãe } & \multicolumn{2}{c}{ Pai } \\
\cline { 2 - 5 } & M & DP & M & DP \\
\hline Interacção Suave & 6,44 & 1,22 & 6,32 & 1,02 \\
Proximidade & 5,63 & 1,14 & 5,08 & 1,09 \\
Contacto Físico & 6,59 & 1,11 & 6,13 & 1,28 \\
Interacção c/ outros adultos & 5,76 & 1,38 & 5,78 & 1,57 \\
\hline
\end{tabular}

Com base numa análise de variância, compararam-se as escalas do $A Q S$ entre mãe e pai, verificando-se a existência de diferenças significativas nas escalas de Proximidade $(F(110,1)=6,63 ; p<0,01)$ e de Contacto Físico $(F(110,1)=4,12 ; p<0,05)$. Como se pode observar, na figura 1 , as crianças têm tendência para manter uma maior proximidade com a mãe, quer seja seguindo-a, ficando perto dela e/ou vigiando a sua localização, bem como para procurar maior contacto físico com a mãe, retirando prazer e conforto do mesmo, comparativamente com o pai.

Não foram encontradas diferenças significativas entre mãe e o pai nas escalas de Interacção Suave $(F(110,1)=0,30 ; p>0,05)$ e de Interacção com Outros Adultos $(F(110,1)=0,03 ; p>0,05)$. Ou seja, nem a qualidade das interacções consideradas harmoniosas, nas quais as crianças partilham e participam num dar e receber afectivo, nem a qualidade das interacções com os outros adultos diferencia as crianças nas suas relações com ambos os pais. 
Utilizando o Coeficiente de Correlação de Pearson (R), correlacionou-se o valor de segurança da criança relativamente à mãe e ao pai com as escalas de Posada e Waters (cit. por Posada, Waters et al., 1995), sendo os resultados apresentados na Tabela 3.

Figura 1: Escalas de Proximidade e de Contacto Físico para mãe e pai

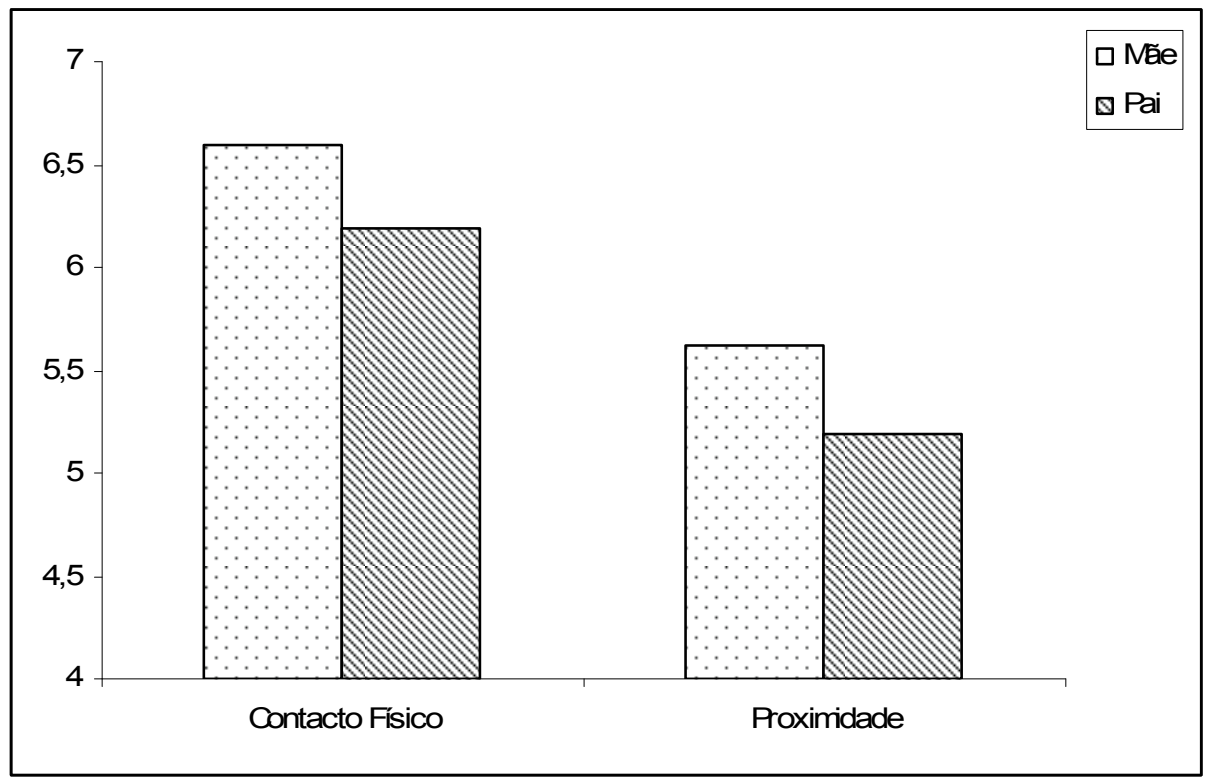

Tabela 3: Correlações entre os valores de segurança relativamente à mãe e ao pai $\mathrm{e}$ as escalas do A.Q.S.

\begin{tabular}{lcccc}
\hline & $\begin{array}{c}\text { Interacção } \\
\text { Suave }\end{array}$ & Proximidade & $\begin{array}{c}\text { Contacto } \\
\text { Físico }\end{array}$ & $\begin{array}{c}\text { Interacção } \\
\text { Outros Adultos }\end{array}$ \\
\hline Segurança/mãe & $0,87^{* *}$ & $0,40^{* *}$ & $0,39 * *$ & $0,25^{*}$ \\
Segurança/ pai & $0,85^{* *}$ & $0,48^{* *}$ & $0,49 * *$ & 0,07 \\
\hline
\end{tabular}

Notas: * A correlação é significativa a $p<0,05 . \quad * *$ A correlação é significativa a $p<0,01$.

Como se pode verificar, na Tabela 3, para as mães, as 4 escalas encontram-se correlacionadas com o valor de segurança, sendo a correlação de 0,87 , com a escala de Interacção Suave muito forte. Para o pai, apenas, a escala de Interacção com Outros Adultos não se encontra correlacionada 
com o valor de segurança. Tal como para a mãe, o valor da correlação com a escala de Interacção Suave é muito forte $(\mathrm{r}=0,85)$.

Correlacionaram-se, ainda, as escalas do $A Q S$ de Posada e Waters (1995) para a mãe e o pai, sendo os resultados apresentados na Tabela 4.

Tabela 4: Correlações das escalas do A.Q.S. para mãe e pai

\begin{tabular}{|c|c|c|c|c|c|c|c|c|}
\hline Escalas & $\begin{array}{l}\text { Interac- } \\
\text { ção } \\
\text { Suave/ } \\
\text { mãe }\end{array}$ & $\begin{array}{l}\text { Proximi- } \\
\text { dade/mãe }\end{array}$ & $\begin{array}{l}\text { Contacto } \\
\text { Físicol } \\
\text { mãe }\end{array}$ & $\begin{array}{c}\text { Interacção } \\
\text { Outros } \\
\text { Adultos } \\
\text { /mãe }\end{array}$ & $\begin{array}{c}\text { Inter- } \\
\text { acção } \\
\text { Suave/ } \\
\text { pai }\end{array}$ & $\begin{array}{c}\text { Proximi- } \\
\text { dade/ } \\
\text { pai }\end{array}$ & $\begin{array}{l}\text { Contacto } \\
\text { Físico/pai }\end{array}$ & $\begin{array}{c}\text { Interacção } \\
\text { Outros } \\
\text { Adultos/pai }\end{array}$ \\
\hline $\begin{array}{l}\text { Interacção } \\
\text { Suave / mãe }\end{array}$ & & & & & & & & \\
\hline $\begin{array}{l}\text { Proximidade/ } \\
\text { mãe }\end{array}$ & 0,19 & & & & & & & \\
\hline $\begin{array}{l}\text { Contacto } \\
\text { Físico/mãe }\end{array}$ & 0,03 & $0,66^{\star *}$ & & & & & & \\
\hline $\begin{array}{l}\text { Interacção } \\
\text { Outros } \\
\text { Adultos/mãe }\end{array}$ & 0,19 & $-0,20$ & $-0,20$ & & & & & \\
\hline $\begin{array}{l}\text { Interacção } \\
\text { Suave/pai }\end{array}$ & $0,27^{*}$ & 0,06 & $-0,05$ & 0,00 & & & & \\
\hline $\begin{array}{l}\text { Proximidade/ } \\
\text { pai }\end{array}$ & 0,04 & $0,35^{\star *}$ & 0,15 & $-0,17$ & 0,19 & & & \\
\hline $\begin{array}{l}\text { Contacto } \\
\text { Físico/pai }\end{array}$ & 0,05 & 0,25 & 0,16 & $-0,22$ & 0,21 & $0,62^{* *}$ & & \\
\hline Interacção & & & & & & & & \\
\hline $\begin{array}{l}\text { Outros } \\
\text { Adultos/pai }\end{array}$ & 0,20 & $-0,27^{*}$ & $-0,24$ & $0,48^{* *}$ & 0,07 & $-0,43^{\star *}$ & $-0,43^{* *}$ & \\
\hline
\end{tabular}

Como se pode verificar, na Tabela 4 , tanto na relação com a mãe, como com o pai, quanto mais elevados os valores nas escalas de Proximidade, mais elevados os valores na escala de Contacto Físico. Por outro lado, quanto mais elevado o valor na escala de Interacção com Outros Adultos/pai, menor os valores nas escalas de Contacto Físico e Proximidade com o pai. Verifica-se, ainda, que quanto maior o valor na escala de Interacção com Outros Adultos/pai, menor é a Proximidade Física relativamente à mãe. Observa-se, também, que, quanto mais elevados os valores nas escalas de Interacção Suave, Proximidade e Interacção com Outros Adultos apresentados para a mãe, mais elevados os valores das mesmas escalas em relação ao pai. 
Análise da concordância ou discordância na organização dos comportamentos de base segura da criança face à mãe e ao pai

Com base no Coeficiente de Correlação de Pearson (R), verificou-se que o valor de segurança da criança face à mãe se encontra significativamente correlacionado com o valor de segurança ao pai $(r=0,35 ; p<0,05)$. Assim, as crianças com um valor de segurança mais elevado face à mãe apresentam, também, um valor de segurança mais elevado relativamente ao pai. Os dados obtidos confirmam os resultados de Veríssimo et al. (2006) para uma amostra portuguesa, de crianças em idade pré-escolar, onde se obteve uma correlação de 0,29 $(p<0,05)$ entre os valores de segurança da mãe e do pai.

\section{Discussão}

O presente estudo analisa o fenómeno de base segura, aspecto central na teoria da vinculação de Bowlby/Ainsworth (Waters \& Cummings, 2000; Waters, 2002), em contexto familiar, centrando-se nas relações mãe/criança e pai/criança. Os resultados obtidos, em meio naturalista e não ansiogénico indicam que as crianças, numa amostra portuguesa, entre os 2 anos e meio e os 3 anos, utilizam a mãe e o pai como base segura, confirmando a noção de que este fenómeno está presente em crianças provenientes de diferentes grupos culturais (Bowlby, 1982; Posada, Goa et al., 1995). O valor médio da segurança para a mãe é de 0,45 e para o pai de 0,41 , os quais se encontram dentro dos valores médios dos estudos apresentados na meta-análise de van IJzendoorn et al. (2004). Nesta amostra, não foram, ainda, encontradas diferenças significativas entre os valores de segurança da criança à mãe e ao pai. Assim, os dados obtidos, com base em informações prestadas por observadores independentes confirmam e alargam os resultados obtidos via self report referidos na meta-análise de van IJzendoorn (2004) que indicam que o valor médio de segurança na relação com o pai é comparável ao da mãe. Refira-se que, nos estudos que utilizam a Situação Estranha (e.g. Main \& Weston, 1981; Rosen \& Rohbaum, 1993), a classificação das crianças na relação com o pai é comparável à referida por Ainsworth et al. (1978) para a relação criança/mãe.

Assim, embora Bowlby e Ainsworth considerem que, em contextos normais, a mãe é a principal figura de vinculação, os valores obtidos não revelam diferenças, numa fase em que a relação pai/criança parece ser particularmente saliente (Easterbrooks \& Goldberg, 1984; Lamb, 1977). Há, contudo, que interpretar estes resultados com cautela, uma vez que as observações foram realizadas de modo independente para a interacção criança/mãe e criança/pai e não na presença de ambas as figuras. Contudo, 
Lamb (1977) verificou que crianças observadas, em casa, durante o primeiro ano de vida, com ambos os pais, não apresentavam preferência por nenhum deles na exibição de comportamentos vinculativos. $\mathrm{O}$ mesmo autor referindo-se a diversas observações, realizadas com outras amostras, afirma que quer o pai, quer a mãe interagem mais com os filhos quando estão sozinhos com eles, do que quando ambos estão presentes, referindo, ainda, que as crianças eram mais activas em situações diádicas, do que triádicas.

Os resultados obtidos confirmam a noção de Bowlby (1982) de que o uso de figuras de vinculação como base segura se mantém central na organização dos comportamentos da criança durante o segundo e a maior parte do terceiro ano. Outras tarefas importantes em termos de desenvolvimento surgem nesta fase, como a progressiva aquisição de autonomia e independência, que segundo Sroufe, Fox e Pancake (1983), são construídas com base na existência de uma relação de vinculação segura na infância. É a utilização da mãe/pai como base segura, que permite à criança, progressivamente, explorar e aprender acerca do seu ambiente, uma vez que está confiante que, em caso de necessidade, a mãe/pai estará acessível e será sensível às suas necessidades (Ainsworth et al., 1978; Bowlby, 1982).

Ao considerar a vinculação enquanto um sistema, é a organização dos comportamentos e não os comportamentos discretos que são importantes para definir o modo como a criança utiliza a mãe ou outra figura como base segura (Sroufe \& Waters, 1977) e que, no caso do $A Q S$, é sintetizado no valor de segurança. Contudo, uma vez que a metodologia $Q$-set permite uma análise a nível de escalas, procurámos, ainda, analisar os aspectos comuns e as especificidades na organização destes comportamentos na relação com a mãe e o pai. Verifica-se que, para ambas as figuras parentais, os comportamentos relacionados com uma interacção suave, com a proximidade e contacto físico estão significativamente correlacionados com os valores de segurança. Ambas as relações são, assim, marcadas por uma comunicação harmoniosa e calorosa, em que as crianças partilham e participam num dar e receber afectivo, sugerindo uma relação mutuamente satisfatória entre a criança e a mãe/pai, elemento característico de uma organização segura dos comportamentos. Característico do fenómeno de base segura é, ainda, o regressar periodicamente para junto da mãe/pai ou o manter a noção da sua localização, podendo optar por ficar mais perto ou afastar-se enquanto explora o ambiente, sabendo que poderá regressar para próximo da mãe/pai quando aflita, aborrecida ou necessitar de ajuda, e que será reconfortada pela mãe/pai.

Embora estando presentes em ambas as relações, os comportamentos relacionados com a proximidade e o contacto físico parecem ser mais salientes na relação com a mãe, com a qual a criança tem tendência a manter uma maior proximidade e contacto físico, comparativamente, com o pai. 
De acordo com a teoria da vinculação, diferentes comportamentos por parte da mãe/pai podem atingir os mesmos objectivos, ou seja, responder de um modo adequado às necessidades da criança; também, para a mesma figura, os comportamentos podem variar de acordo com os contextos e situações, mas os resultados para a criança são os mesmos. Do ponto da vista da criança, o importante é que esta aprenda que a mãe/pai é responsiva e sensível aos seus sinais e que está disponível quando ela necessita (Posada, Jacobs, Carbonell, Alzate, Bustamante \& Arenas, 1999).

$\mathrm{Na}$ amostra em estudo, os comportamentos relacionados com a interacção com outros adultos não apresentam diferenças significativas para a mãe e para o pai, embora se encontrem apenas correlacionados com a segurança em relação à mãe. As interacções com estranhos poderão ter as suas raízes mais no sistema social, do que no sistema de vinculação. Com efeito, os estudos empíricos que relacionam a vinculação com a interacção com estranhos são menos consistentes do que, por exemplo, com a interacção com os pares (Berlin \& Cassidy, 1999). Bridges et al. (1998) verificaram que o comportamento das crianças com os pais, mas não com as mães, previa as subsequentes interacções da criança com o estranho, na Situação Estranha. Contrariamente, os resultados obtidos por Main e Weston (1981) indicam que a associação entre a segurança da relação face à mãe e a predisposição da criança para estabelecer uma relação amigável com um adulto é mais forte, comparativamente, com a relação face ao pai.

Os dados vão, assim, ao encontro do descrito na literatura de que mãe e pai, funcionando como base segura, apresentam diferenças nos seus estilos de interacção (Lamb, 1977; Lewis \& Lamb, 2003), o que poderá promover determinadas características ou comportamentos da criança. Por outro lado, a mãe/pai poderão estimular comportamentos ou características da criança que estão relacionados com os seus valores parentais e que não estão, necessariamente, relacionados com a vinculação, mas com a sociabilidade e o temperamento (Fox et al., 1991; Steele et al., 1996).

Relativamente à analise da concordância ou não dos valores de segurança, à mãe e ao pai, verifica-se uma correlação de $0,35(p<0,05)$, o que vem confirmar os resultados obtidos por Veríssimo et al. (2006) e vai ao encontro de outros estudos (e.g., Caldera, 2004; Frosch et al., 2000; Rosen \& Rothbaum, 1993; Rosen \& Burke, 1999; Steele et al., 1996). Na meta-análise realizada por van IJzendoorn e De Wolff (1997), encontrou-se apenas uma correlação modesta $(r=0,17)$ entre a qualidade da vinculação da criança à mãe e ao pai, pelo que, para os autores, a segurança não parece estar substancialmente generalizada às diferentes relações dentro do contexto familiar. Assim, a segurança da vinculação deve ser vista como uma característica da relação e não é um traço da criança (Ainsworth et al., 1978; Bowlby, 1982; Main \& Weston, 1981; Sroufe \& Waters, 1977; Sroufe, 1985). 
Há, pois, que analisar os resultados com algum cuidado. A concordância obtida na amostra em estudo pode ser resultado da semelhança dos cuidados parentais, ou seja, se ambas as figuras são sensíveis e responsivas aos sinais da criança, como se pode inferir pela organização dos comportamentos das crianças, será de esperar uma concordância nas classificações da criança relativamente à mãe e ao pai (Cassidy, 1999; Fox et al., 1991). Assim, esta semelhança nos comportamentos parentais poderá ser explicada pela semelhança das representações de vinculação entre o casal (van IJzendoorn \& Bakermans-Kranenburg's, 1996). Poder-se-á colocar, ainda, a hipótese de um pai servir de modelo ao outro, ou ambos poderem aprender em conjunto a responder de um modo sensível (ou insensível) à criança (Belsky et al., 1984; Fox et al., 1991; Sagi et al., 1995). Por outro lado, um progenitor, nomeadamente a mãe, poderá ainda influenciar a natureza da relação que a criança tem com o outro progenitor (Steele et al., 1996). Outra explicação poderá residir nas características individuais da criança, nomeadamente o temperamento (e.g., Fox et al., 1991; Cassidy, 1999).

Segundo Sroufe e Waters (1977), a segurança da vinculação reflecte a qualidade da relação especifica entre a criança e a mãe/pai, pelo que nela são incluídas a história das interacções diádicas entre a criança e a mãe/pai, características da criança e da mãe/pai e o contexto em que essa díade se insere.

\section{Referências}

Ainsworth, M. D. S. (1967). In Infancy in Uganda - chapter 20. Retrieved (2002) from http://www.psychology.sunysb.edu/attachment/ ainsworth/ains-worth_index.

Ainsworth, M. D. S. (1989). Attachments beyond infancy. American psychologist, 44, 709-716.

Ainsworth, M. D. S., Blehar, M., Waters, E., \& Wall, S. (1978). Patterns of attachment: A psychological study of the strange situation. Hillsdale, N. J.: Erlbaum.

Belsky, J., Gilstrap, B., \& Rovine, M. (1984). The Pennsylvania infant and family development project I: Stability and change in mother-infant and father-infant interactions in a family setting at one, three and nine months. Child Development, 55, 692-705.

Belsky, J., Rovine, M., \& Taylor, D. (1984). The Pennsylvania infant and family development project: Origins of individual differences in infant-mother attachments: Maternal and infant contributions. Child Development, 55, 718-728 .

Berlin, L. J., \& Cassidy, J. (1999). Relations among relationships. Contributions from attachment, theory and research. In J. Cassidy \& P. R. Shaver (Eds.), Handbook of attachment: Theory, research, and clinical applications (pp. 688-711). New York: Guilford. 
Bowlby, J. (1973). Attachment and Loss: Vol. 2. Separation, anxiety, and anger. New York: Basic.

Bowlby, J. (1982). Attachment and loss: Vol. 1. Attachment (2d Rev. Ed.) New York: Basic Books (Original work published, 1969).

Bowlby, J. (1989). The role of attachment in personality development and psychopathology. In S. Greenspan \& G. Pollack (Eds.), The course of life. Vol. 1: Infancy (pp. 229-270). Madison Connecticut: International University Press.

Bowlby, J. (2002). Cuidados Maternos e Saúde Mental. São Paulo: Martins Fontes (4. ${ }^{\mathrm{a}}$ Ed.)

Bretherton, I. (1992). The origins of the attachment theory: John Bowlby and Mary Ainsworth. Developmental Psychology, 28 (5), 759-775.

Bridges, L., Connell, J. P., \& Belsky, J. (1988). Similarities and differences in infant-mother and infant-father interactions in the strange situation: A component process analysis. Developmental Psychology, 24 (1), 92-100.

Cabrera, N. J., Tamis-LeMonda, C. S., Bradley, R. H., Hofferth, S., \& Lamb, M. (2001). Fatherhood in the twenty-first century. Child Development, 71 (1), 127 $-136$.

Caldera, Y. M. (2004). Paternal involvement and infant-father attachment: A Q-set study. Fathering, 2 (2), 191-210.

Cassidy, J. (1999). The nature of child's ties. In J. Cassidy \& P. R. Shawer (Eds.), Handbook of attachment theory. Research and clinical applications (pp. 3-20). New York: The Guilford Press.

Cox, M., Owen, M., Henderson, V., \& Margand, N. (1992). Prediction of infant-father and infant-mother attachment. Developmental Psychology, 28 (3), 474-483 .

Cowan, P. A. (1997). Beyond meta-analysis: A plea for a family systems views of attachment. Child Development, 68, 601-603.

Easterbrooks, M. A., \& Goldberg, W. A. (1984). Toddler development in the family: Impact of father involvement and parenting characteristics. Child Development, $55,740-752$.

Fox, N., Kimmerly, N., \& Schafer, W. (1991). Attachment to mother/attachment to father: A meta-analysis. Child development, 62, 210-225.

Frosch, C., Mangelsdorf, S. C. \& McHale, J. L. (2000). Marital behaviour and the security. Journal of Family Psychology, 14 (1), 144-161.

Goossens, F. A., \& Van Ijzendoorn, M. H., (1990). Quality of infants' attachments to professional caregivers. Relation to infant-parent attachment and day-care characteristics. Child Development, 61, 832-837.

Grossmann, K., Grossmann, K., Fremmer-Bombik, E., Kindler, H., Scheuerer-Englisch, H., \& Zimmermann, P. (2002). The uniqueness of the child-father attachment relationship: Father's sensitive and challenging play as a pivotal variable in a 16-year longitudinal study. Social development, 11 (3), 307-331.

Lamb, M. (1977). Father-infant and mother-infant interactions in the first year of life. Child Development, 48, 167-181. 
Lamb, M. (1987). Introduction: The emergent American father. In M. Lamb (Ed.), The father's role: Cross-cultural perspectives (pp. 3-26). New Jersey: Lawrence Erlbaum Associates.

Lamb, M., Frodi, M., Hwang, C., \& Frodi, A. (1983). Effects of paternal involvement for mothers and fathers. Child Development, 54, 450-458.

Lewis, C., \& Lamb, M. (2003). Fathers' influences on children's development: The evidence from two-parents families. European Journal of Psychology of Education, 18 (2), 211-228.

Main, M., \& Weston, D. R. (1981). The quality of the toddler's relationship to mother and to father: Related to conflict behaviour and the readiness to establish new relationships. Child Development, 52, 932-940.

Marvin, R. S., \& Britner, P. A. (1999). Normative development: The ontogeny of attachment. In J. Cassidy \& P. R. Shaver (Eds.), Handbook of attachment theory: Research and clinical applications (pp. 44-67). New York: The Guilford Press.

NICHD Early Child Care Research Network (1997). The effects of infant child care on infant-mother attachment security: Results of the N.I.C.H.D. Study of Early Child Care. Child Development, 68 (5), 860-879.

Posada, G., Goa, Yuan, Wu, Fang, Posada, R., Tascon, M., Schoelmerich, A., Sagi, A., Kondo-Ikemura, K., Haaland, W., \& Synnevaag, B. (1995). The secure-base phenomenon across cultures: Children's behaviour, mother's preferences and experts concepts. In Waters, Vaughn, Posada \& Kondon-Ikemura (Eds.), Monographs of the Society for Research in the Child Development, 60 (2-3), 27-47.

Posada, G., Jacobs, A., Carbonell, G., Alzate, G., Bustamante, M., \& Arenas, A. (1999). Maternal care and attachment security in ordinary and emergency contexts. Developmental Psychology, 35 (6), 1379-1388.

Posada, G., Waters, E., Crowell, J. A., \& Lay, K. (1995). Is it easier to use a secure mother as a secure base? Attachment Q-Sort correlates of adult attachment interview. In Waters, Vaughn, Posada \& Kondon-Ikemura (Eds.), Monographs of the Society for Research in the Child Development, 60 (2-3), 133-145.

Rosen, K. S., \& Burke, P. B. (1999). Multiple attachment relationships within families: Mothers and fathers with two young children. Developmental Psychology, 35 (2), 436-444.

Rosen, K. S., \& Rothbaum, F. (1993). Quality of parental caregiving and security of attachment. Developmental Psychology, 29 (2), 358-367.

Sagi, A., Van IJzendoorn, M. H., Aviezer, O., Donnell F., Koren-Karie, N. Joels, T., \& Harel, Y. (1995). Attachments in a multiple-caregiver and multiple-infant environment: The case of the Israeli Kibbutzim. In Waters, Vaughn, Posada \& Kondon-Ikemura (Eds.), Monographs of the Society for Research in the Child Development, 60 (2-3), 71-91.

Sroufe, A. (1985). Attachment classification from the perspective of infant-caregiver relationships and infant temperament. Child Development, 56, 1-14.

Sroufe, A., Fox, N. E., \& Pancake, V. R. (1983). Attachment as an organizational construct. Child Development, 48, 1184-1199. 
Sroufe, A., \& Waters, E. (1977). Attachment and dependency in developmental perspective. Child Development, 54, 1615-1627.

Steele, H., Steele, M., \& Fonagy, P. (1996). Associations among attachments classifications of mothers, fathers and their infants. Child Development, 67, 541-555.

Thompson, R. A. (1998). Early sociopersonality development. In W. Damon \& N. Eisenberg (Eds.), Handbooks of Child Psychology - Social, Emotional and Personality Development: New york: Ed. John Wiley \& Sons.

Van IJzendoorn, M. H., \& Bakermans-Kranenburg, M. J. (1996). Attachment representations in mothers, fathers, adolescents, and clinical groups: A meta-analytic search for normative data. Journal of Consulting and Clinical Psychology, 64, $8-27$.

Van IJzendoorn, M. H., \& De Wolf, M. (1997). In search of the absent father-meta-analyses of infant-father attachment: a rejoinder to our discussants. Child Development, 68, 604-609.

Van IJzendoorn, M. H., Vereijken, C. M., Bakermans-Kranenburg, M. J., \& Riksen-Walraven, J. M. (2004). Assessing attachment security with the attachment Q-sort: Meta-analytic evidence for the validity of the observer AQS. Child Development, 75, 1188-1213.

Veríssimo, M., Blicharsky, T., Strayer, F., \& Santos, A. (1995). Vinculação e estilos de comunicação da criança. Análise Psicológica, 1-2 (XIII), 145-155.

Veríssimo, M., Monteiro, L., \& Santos, A. J. (2006). Para além da mãe: vinculação na tríade mãe-pai-criança. In J.C. Coelho Rosa \& S. Sousa (Eds.), Caderno do bebé (pp. 73-85). Fim de Século.

Veríssimo, M., Monteiro, L, Vaughn, B., Santos, A. J., \& Watters, H. (2005). Coordenação entre o modelo interno dinâmico da mãe e o comportamento de base-segura dos seus filhos. Análise Psicológica, XXIII (2), 7-17.

Waters, E. (1995). Appendix A: Attachment Q-set (version 3.0). In Waters, Vaughn, Posada \& kondon-Ikemura (Eds.), Monographs Child development, 60 (2-3), 234-246.

Waters, E. (2002). Live long and prosper. Retrieved (2006) from http://www.psychology.sunysb.edu/attachment/gallery/live_long_/live_long.ht $\mathrm{ml}$.

Waters, E., \& Cummings, E. M. (2000). A secure base from which to explore close relationships. Child Development, 71, 164-172.

Waters, E., \& Deane, K. (1985). Defining and assessing individual differences in attachment relationships: Q-methodology and the organization of behavior in infancy and early childhood. In I. Bretherton \& E. Waters (Eds.), Monographs of the Society for Research in the Child Development, 50 (1-2), 41-65.

Waters, E., Noyes, D., Vaughn, B., \& Ricks, M. (1985). Q-sort definitions of social competence and self-esteem: Discriminant validity of related constructs in theory and data. Developmental Psychology, 21, 508-522. 